ORIGINAL ARTICLE

\title{
Subtle Clinical Manifestation in SLE: Clinically and Laboratory Diagnosis and Follow Up
}

\author{
EDWARD KURNIA SETIAWAN LIMIJADI', LISYANI B. SUROMO' \\ ${ }^{1}$ Department of Clinical Pathology, Faculty of Medicine, Diponegoro University, Semarang, Central Java, Indonesia, \\ Corresponding author: Edward Kurnia Setiawan Limijadi, Email: edwardksl@fk.undip.ac.id Phone: +62 83862305904;
}

\section{ABSTRACTS}

Systemic Lupus Erythematosus (SLE) cases in pediatric are uncommon, compared to adult patients, who are mostly undetected at primary healthcare, especially with subtle clinical manifestations. Detection is necessary as early as possible so that further progressivity can be controlled. A 12-year-old child came with a rash on the face confirmed by a physical examination as malar rash and discoid rash. Laboratory results showed a positive antinuclear antibody (ANA) and double-stranded DNA (dsDNA) while complete blood count, liver function test, kidney function test and urinalysis were found within normal limits. Based on those findings we conclude that the diagnosis was SLE with subtle clinical manifestation. Evaluation needs to be done at least every 6 months as a scoring of the activity of disease and organ damage. The evaluation includes signs and symptoms, laboratory work-up such as complete blood count, organ function test, urinalysis, and immunologic parameters.

Keywords: systemic lupus erythematosus, pediatric, subtle clinical manifestation

\section{INTRODUCTION}

Systemic lupus erythematosus (SLE) is an autoimmune disease with clinical manifestations varying from mild to severe. The incidence of SLE is more common in adults than in children. The peak age of affected children is 15 years old with the incidence of women being greater than men which is 9:1. ${ }^{1}$ The most frequent incidence in Indonesia, according to Setiabudiawan, et al. (2017), was found in West Java, North Sumatra, Jakarta, and South Sulawesi. ${ }^{1}$

The cause of SLE is not certainly known and there is usually a predisposing factor such as genetic factor. The presence of autoantibodies and complement deficiencies (C1q, C2, C4) increases the risk of SLE. Sun exposure, hormonal factors, infections and the environment can also triggers the emergence and recurrence of SLE. ${ }^{2}$

\section{CASE REPORT}

A 12-year-old Asian girl came to the dermatovenereology outpatient clinic of the hospital with her mother. She complained of patches in her facial skin that had lasted for approximately 3 weeks. These patches were not itchy, and painless, but multiplied in the face area. The patient did not use facial cream or any other skin products applied to her face before the complaint appeared. There were no complaints related to disturbed vision, joint pain, yellowing of the body skin, enlarged abdomen, abnormal urination and bowel movements. The patient's mother claimed to not give any treatment to the skin of her child's face. The patient had never before experienced a similar disease or other severe illnesses. No other family members of patients had a history of such complaints.

Patients generally appeared conscious with vital signs were normal limits. Examination of the facial area found malar rash and discoid rash (Figure 1). There were no abnormalities in the eyes, nose, mouth and ears. There were no enlarged lymph nodes in the neck or other areas. Examination of the heart, lungs and abdomen were within normal limits, there were no organomegaly. The upper and lower limb had no movement disorders, and could function normally, and there were no abnormalities in the skin of the limbs. Laboratory test showed normal erythrocyte, leukocyte, and platelet profiles. Examination of organ functions were within normal limits. ANA examination found positive results then continued with ANA profile examination. Anti-dsDNA was found to be positive, while other antibody tests had negative results. The following Table 1 showed results of a complete laboratory examination of the patient.

\section{DISCUSSION}

Patient came with main complaint of facial patches, especially in the area around the nose, which was confirmed by a physical examination showing that the spots were malar rash and other spots on the face were in the form of discoid rash. A thorough physical examination found no other abnormalities in the patient's body. The discovery of this situation leads to the diagnosis of cutaneous lupus erythematosus.

Hematological laboratory results found only slightly decreased platelets which was not included in the finding of hematological abnormalities in the diagnosis criteria for SLE according to the American College of Rheumatology (ACR) which was revised in 2012. Clinical chemistry and urinalysis examining liver and kidney function did not show any abnormalities in both organs. The findings of laboratory results that were meaningful were immunological parameters, which were positive ANA and anti-dsDNA. This was consistent with the research of Jakes et al. (2012) that the two parameters were the most positive parameters in systemic lupus erythematosus. ${ }^{3}$

The diagnosis of SLE was based on clinical findings and laboratory results. It is appropriate that clinical presence of malar and discoid rash were found and immunological parameters were found in laboratory results. It is said that these four criteria include one clinical sign and one immunological laboratory result. ${ }^{4}$ Clinical finding in this case was found only on the skin of the face, so that the patient was said to have SLE with minimal clinical manifestations. The course of the disease theoretically can appear on one organ only (minimum lesion), but subsequent developments that affect the organs cannot be 
known regarding when they will appear and these should be known immediately. The condition of the patient's organs needs clinical and laboratory evaluation. Laboratory tests can include complete hematology, clinical chemistry, urinalysis, immunology, and creatinine-albumin ratio. ${ }^{5}$

The Systemic Lupus Erythematosus Disease Activity Index (SLEDAI) is a scoring system in the form of 24 assessment parameters for SLE. This patient was assessed using SLEDAI and got a score of 4 . This score showed low disease activity (inactive) where the reference value was a score of $0-5 .^{6}$ If the score obtained was more than or equal to 6 , according to SLEDAI, the disease in a patient was considered active which could cause damage to organs faster. Another score to assess the presence of organ damage is The Systemic Lupus International Collaborating Clinics/American College of Rheumatology (SLICC/ACR) damage index. This scoring system is carried out on 12 related organs and the damage score assessment should be done at least every 6 months to monitor the progression of the disease. The more scores obtained the worse of the prognosis will be. ${ }^{7}$

The characteristic of SLE is loss of tolerance resulting in auto-reactivation of $T$ and $B$ cells which leads to the production of pathogenic autoantibodies against nucleic acids and proteins in them which produce tissue / organ damage. Tolerance loss occurred due to genetic abnormalities, risks from environmental factors, and the exact time of the emergence of the disease. There are 30 genetic loci that play a role in causing tolerance abnormalities. Immunologically, this disease involves the innate immune system and is obtained by complex pathoimmunology. ${ }^{8}$

Complement deficiency in SLE cases is an important matter to discuss. The case of this patient was likely to have a complement deficiency such as C1q, C1r, C1s, C4, and C2 simultaneously or singly which was the trigger factor for the emergence of SLE. This deficiency could occur due to mutations in protein-forming genes in the complement. The condition of complement deficiency made the activation of the classical and lectin complement pathways disturbed. The process of cell apoptosis opsonization mediated by complement became slower that the process of recognition by $\mathrm{T}$ lymphocyte cells occurred and the possibility of a higher inflammatory process occurred and triggered an autoimmune state. The use of $\mathrm{C} 1 \mathrm{q}$ is that $\mathrm{C} 1 \mathrm{q}$ can provide active protection by regulating auto-reactive immune cells. This is supported by the statement that $\mathrm{C} 1 \mathrm{q}$ inhibits $\mathrm{T}$ cell proliferation in the in vitro experiments. ${ }^{9}$

The presence of one or several trigger factors in genetic predisposing individuals produces abnormal CD4 T cells. This causes loss of cell tolerance to self-antigens, resulting in auto-reactive $\mathrm{T}$ cells appearing which cause induction and expansion of $B$ cells, then produce autoantibodies as well as memory B cells. Autoantibodies not only bind to antigens forming immune complexes result in tissue damage but also complement which eventually bind to the $\mathrm{C} 3 \mathrm{~b}$ receptor in erythrocytes which causes hemolysis. Parameters that can be used to determine the attachment of immune complexes in erythrocytes are C4d, where an increased C4d level along with the high number of immune complexes present on the erythrocyte surface. ${ }^{10}$
Complications of SLE in patients are detected immediately by examining the related organs.

Table 1. Laboratory Examination Results of the Patients

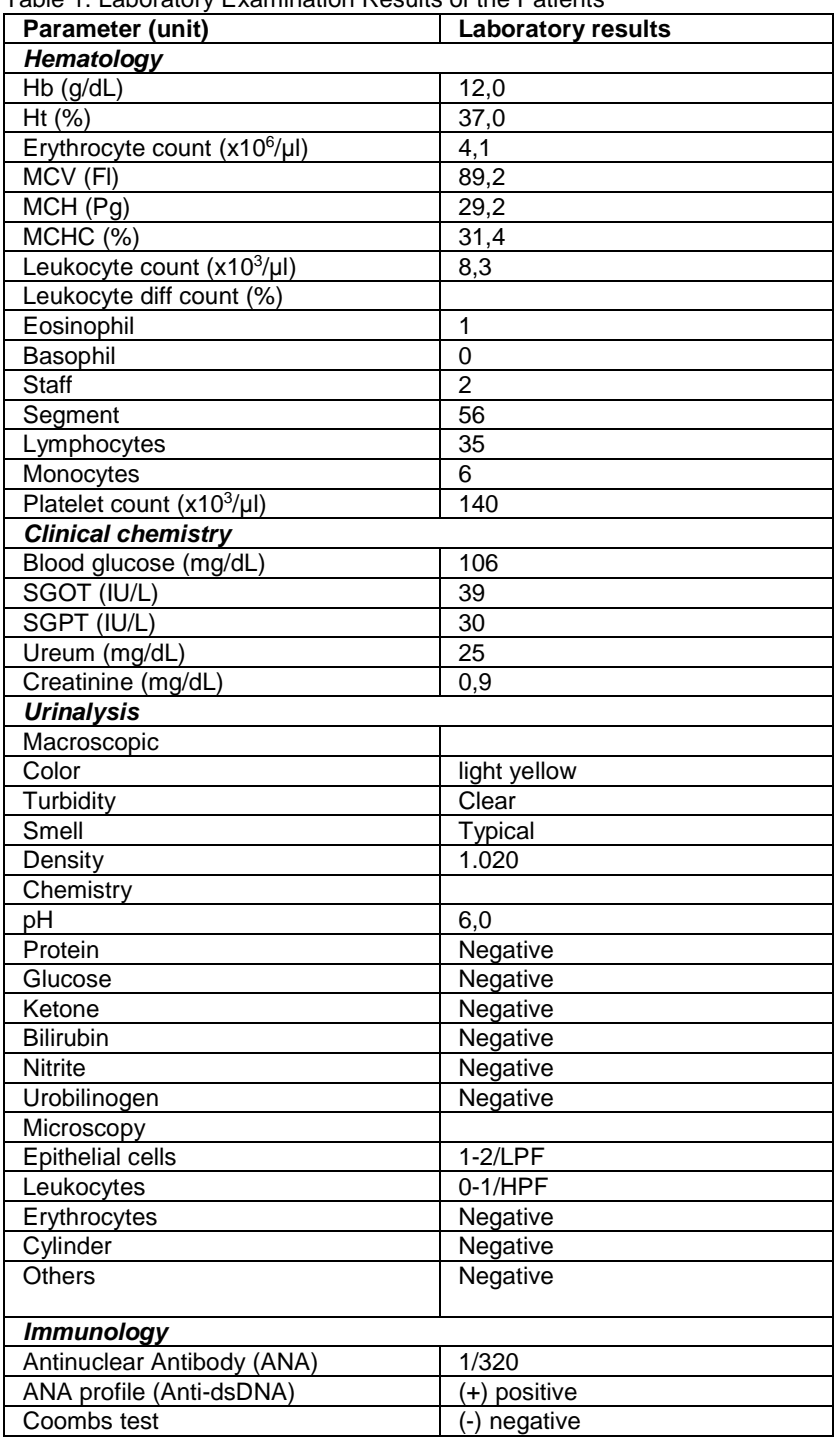

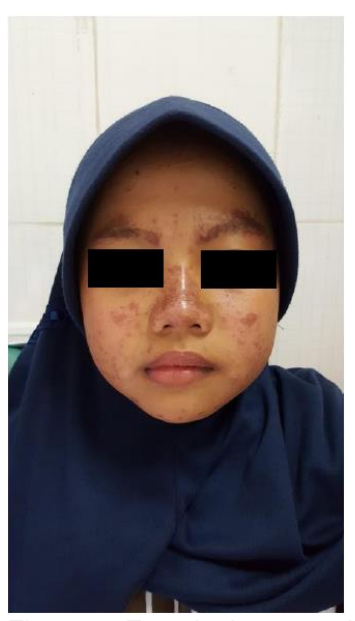

Figure 1. Face lesions consist of malar rash and discoid rash. 


\section{CONCLUSION}

SLE can occur in children even though the incidence is less that in adults. Clinical manifestations can vary in each individual from mild to severe. Laboratory tests are needed to support the diagnosis of SLE and monitoring of disease progressivity in addition to possible developing clinical manifestations.

Acknowledgements: We thank the laboratory technicians at Ajibarang Public Hospital for running the laboratory examination of this patient.

\section{REFERENCES}

1. Setiabudiawan B, Ghrahani R. Indonesian epidemiologic data of paediatric systemic lupus erythematosus. BMJ Journals. 2017. 4(1):A201.

2. George Bertsias, Ricard Cervera, Boumpas DT. Systemic lupus erythematosus: pathogenesis and clinical features. In: EULAR textbook on rheumatic disease; 2012:476-500.

3. Jakes RW, Sang CB, Loutherenoo W, Chi CM, Navarra SV, Kwon N. Systematic Review of the Epidemiology of Systemic Lupus Erythematosus in the Asia-Pacific Region: Prevalence, Incidence, ClinicalFeatures, and Mortality. ACR. 2012. 64(2):159-68.

4. Bartel CM. Muller D, Diamond HS. Systemic lupus erythematosus (SLE) work up. 2017. Available at: https://emedicine.medscape.com/article/332244-workup.

Accessed March 10, 2018.

5. Levy DM, Kamphui S. Systemic Lupus Erythematosus in Children and Adolescents. PediatrClin North Am. 2012; 59(2):345-64.

6. Bertsias G, Cervera R, Boumpas DT. Systemic lupus erythematosus: pathogenesis and clinical features. In: Bijlsma JWJ, Hachulla E, editors. EULAR Textbook on Rheumatic Diseases. England: BMJ Groups. 2015. p.476505.

7. Anic F, Butorac MZ, Stimac D, Novak S. New classification criteria for systemic lupus erythematosus corelate with disease activity. Croat Med J. 2014;55:514-9.

8. Taylor KE, Chung SA, Graham RR, Ortmann WA, Lee AT, Langefeld $C D$, et al.Risk alleles for systemic lupus erythematosus in a large case-control collection and associations with clinical subphenotypes. PLoS Genet. 2011;7:e1001311.

9. Kittlesen DJ, Chianese-Bullock KA, Yao ZQ, Braciale TJ, Hahn YS. Interaction between complement receptor gC1qR and hepatitis $\mathrm{C}$ virus core protein inhibits T-lymphocyte proliferation. J Clin Invest. 2000;106(10):1239-49.

10. El Halim HA, Salah M, Ismail W, Fathy A, Ramadhan H. Erythrocyte and glomerular C4d deposits as a biomarker for active lupus nephritis. Egyptian rheumatologist. 2017;1-5. 